\title{
Approximate scaling relation for the anharmonic electron-phonon problem
}

\author{
J. K. Freericks ${ }^{1}$, V. Zlaticí ${ }^{1,2}$, and M. Jarrell ${ }^{3}$ \\ ${ }^{1}$ Department of Physics, Georgetown University, Washington, DC 20057-0995 \\ ${ }^{2}$ Institute of Physics, Zagreb, Croatia \\ ${ }^{3}$ Department of Physics, University of Cincinnati, Cincinnati, OH 45221
}

(December 4, 2017)

An approximate scaling relation is found for the transition temperature to a charge-density-wave instability in the anharmonic electron-phonon problem, which maps a wide range of interaction strengths, anharmonicities, and phonon frequencies onto a common functional form. The relation employs the wave-function renormalization parameter and is valid even for systems that are not Fermi liquids.

Introduction The interaction of electrons with anharmonic lattice vibrations is a long-standing problem that is not yet fully understood. What is surprising about this problem is that nearly all real materials are anharmonic (as can be seen by the fact that they expand or contract upon heating), but quasiharmonic models (which replace the anharmonic phonons by harmonic phonons with temperature-dependent phonon frequencies) work remarkably well at describing properties of most materials [1]. Superconductivity is described most accurately, where the theory of electrons interacting with harmonic phonons, introduced by Migdal [2] and Eliashberg [3], can routinely reproduce experimental tunneling conductances to better than one part in a thousand. The explanation for this result is actually quite simple - the thermal effects that arise due to a nonuniform spacing of the anharmonic energy levels, are unimportant when the temperature is much less than the effective phonon frequency (defined by the difference in energy between the ground and the first-excited state of the anharmonic phonon) [4]. Furthermore, quantum Monte Carlo (QMC) studies [5], have shown that anharmonicity does not appear to produce any exotic behavior, such as enhancements of transition temperatures, or novel superconducting behavior [6]. Instead, the results indicate that the main effect of anharmonicity is to generically break particle-hole symmetry. The discovery we present here is that the anharmonic systems can be mapped onto harmonic ones, with results from widely different parameter regimes collapsing onto the same scaling curve. We believe that this result sheds light onto the question of

(C) 1999 by the authors. Reproduction of this article by any means is permitted for non-commercial purposes. why harmonic models work so well for describing properties of real materials.

Our strategy is to solve anharmonic models in the limit of large spatial dimension [7] where the lattice manybody problem can be mapped onto a self-consistently embedded impurity problem that is solved via a QMC simulation [8] for quantum phonons, or via an iterative transcendental equation for classical phonons [9, 10].

Model The simplest electron-phonon model that includes anharmonic effects is the anharmonic Holstein model 11] in which the conduction electrons interact with local phonon modes:

$$
\begin{aligned}
H & =-\sum_{i, j, \sigma} t_{i j} c_{i \sigma}^{\dagger} c_{j \sigma}+\sum_{i}\left(g \bar{x}_{i}-\bar{\mu}\right)\left(n_{i \uparrow}+n_{i \downarrow}\right) \\
& +\frac{1}{2 M} \sum_{i} p_{i}^{2}+\frac{1}{2} \bar{\kappa} \sum_{i} \bar{x}_{i}^{2}+\alpha_{a n} \sum_{i} \bar{x}_{i}^{4} .
\end{aligned}
$$

Here, $c_{i \sigma}^{\dagger}\left(c_{i \sigma}\right)$ creates (destroys) an electron at site $i$ with spin $\sigma, n_{i \sigma}=c_{i \sigma}^{\dagger} c_{i \sigma}$ is the electron number operator, $\bar{\mu}$ is the chemical potential, and $\bar{x}_{i}\left(p_{i}\right)$ is the phonon coordinate (momentum) at site $i$. The hopping of electrons is restricted to nearest-neighbor lattice sites $i$ and $j$ on a $d$-dimensional hypercubic lattice. $t_{i j}$ is isotropic with magnitude $t=: t^{*} / 2 \sqrt{d}$ where $t^{*}=1$ to define the energy scale. The bare density of states then becomes a Gaus$\operatorname{sian} \exp \left(-\epsilon^{2}\right) / \sqrt{\pi}$. The local phonon has a mass $M$ and a spring constant $\bar{\kappa}$ associated with it. The anharmonic contribution to the phonon potential energy is chosen to be a quartic in the phonon coordinate with a strength $\alpha_{a n}$. The deformation potential (electron-phonon interaction strength) is parameterized by an energy per unit length $g$.

A shift of the phonon coordinate is useful for calculations, and for illustrating the particle-hole symmetry of the model. We shift $\bar{x}_{i}=: x_{i}+x^{\prime}$, with $g+\bar{\kappa} x^{\prime}+3 \alpha_{a n} x^{\prime 3}=$ 0 , to transform the Hamiltonian into

$$
\begin{aligned}
H & =-\sum_{i, j, \sigma} t_{i j} c_{i \sigma}^{\dagger} c_{j \sigma}+\sum_{i}\left(g x_{i}-\mu\right)\left(n_{i \uparrow}+n_{i \downarrow}-1\right) \\
& +\frac{1}{2 M} \sum_{i} p_{i}^{2}+\frac{1}{2} \kappa \sum_{i} x_{i}^{2}+\beta_{a n} \sum_{i} x_{i}^{3}+\alpha_{a n} \sum_{i} x_{i}^{4}
\end{aligned}
$$

with $\kappa:=\bar{\kappa}+12 \alpha_{a n} x^{\prime 2}, \beta_{a n}:=4 \alpha_{a n} x^{\prime}$, and $\mu:=\bar{\mu}-g x^{\prime}$. It is the presence of the cubic term when $\alpha_{a n} \neq 0$ that removes particle-hole symmetry from the problem when $\mu=0$, since the particle-hole transformation is $x_{i} \rightarrow-x_{i}$ 
and $n_{i \sigma} \rightarrow 1-n_{i \sigma}$. The system does continue to possess nesting at half-filling, though, which implies that it will have a nonzero transition temperature to a chargedensity-wave (CDW) at half-filling for any nonzero interaction strength $g$.

We examine two different cases in this contribution: quantum phonons with a large enough phonon frequency that vertex corrections are important $(\Omega=\sqrt{\kappa / M}=0.5$, corresponding to approximately one eighth the effective bandwidth) and classical phonons with zero phonon frequency $(M \rightarrow \infty$ and $\Omega=0)$. The former problem is solved using QMC techniques that have been described elsewhere [5,8]. Transition temperatures are determined by calculating the relevant susceptibility (in this case to a chessboard-phase CDW), and determining the temperature where it diverges (when Trotter error is important, we extrapolate $T_{c}$ using $\Delta \tau=0.2$ and 0.4 , otherwise, we use the larger $\Delta \tau$ ). In the latter case, the problem is solved within the ordered phase, employing a generalization of the Brandt-Mielsch formalism to the static Holstein model [9, 10], and determining $T_{c}$ as the highest temperature that sustains long-range order.

Results We begin in Fig. 1(a) by showing the transition temperature to the commensurate CDW at half filling for the harmonic case, and three different phonon frequencies, $\Omega=0, \Omega=0.5$, and $\Omega=\infty$ which is identical to the attractive Hubbard model [5, 12]. The symbols are the exact, or QMC results (the dotted lines are guides to the eye), while the other lines are second-order weak-coupling conserving approximations [13] (dashed) and second-order strong-coupling calculations 14 (solid). Notice how the maximal $T_{c}$ is essentially independent of phonon frequency, and that the phase diagrams are not too sensitive to phonon frequency when $\Omega$ is smaller than the bandwidth. However, note that the classical phonon case $(\Omega=0)$, and the quantum phonon case are quite different, since $T$ is always above $\Omega$ for the classical phonons, but $T<\Omega$ for the quantum phonon cases shown here. The similarity in $T_{c}$ is surprising, because the CDW vertex is strongly temperature dependent for the quantum phonons, with it's magnitude changing between $-2 g^{2} / \kappa$ and $-g^{2} / \kappa$ as the temperature and frequency are varied.

The anharmonicity is turned on in Fig. 1(b) for similar effective electron-electron coupling strengths and $\Omega=0$ and $\Omega=0.5$. The infinite-phonon frequency limit is unaffected by the anharmonicity, since the phonons respond instantaneously to the electrons, and remain at the origin. We plot the strength of the anharmonicity as $\alpha_{a n} \bar{x}^{* 4}$, where $\bar{x}^{*}$ is the equilibrium coordinate of the atomic system with one electron per site, found from Eq. (11) with $t_{i j}=0$. In the case of weak anharmonicity, this equilibrium coordinate lies near $-g / \kappa$ and the anharmonic energy grows linearly with $\alpha_{a n}$, but as the anharmonicity becomes large, then $\bar{x}^{*}$ lies near $-\left(g / \alpha_{a n}\right)^{1 / 3}$, and the anharmonic energy decreases as $\alpha_{a n}^{-1 / 3}$. This is why the curves in Fig. (1(b) are multivalued for some values of the interaction strength. Notice that the results for different phonon frequencies are similar in qualitative behavior, but that the quantitative results can differ by large amounts as the anharmonicity increases.

We find that the $T_{c}$ satisfies an approximate scaling relation when we plot it instead as a function of $1 / Z(0)$, the wave-function renormalization parameter, in Fig. 2. Nearly two-hundred data points collapse onto the same scaling curve. But the scaling is only approximate, because the infinite-phonon frequency limit, lies well off the scaling curve for low-to-moderate phonon frequencies, so this result must break down as $\Omega$ increases (note, however, that the value $\Omega / t^{*}=0.5$ is larger than the phonon frequency in nearly all real materials). The other lines in Fig. 2 correspond to different weak-coupling approximations plotted now as a function of $Z(0)$ rather than of $U$. The wave function renormalization parameter $Z(0)$ is extracted from the calculations by a linear extrapolation along the Matsubara frequency axis. We compute

$$
Z(0)=1-\frac{3}{2} \frac{\operatorname{Im} \Sigma(i \pi T)}{\pi T}+\frac{1}{2} \frac{\operatorname{Im} \Sigma(3 i \pi T)}{3 \pi T},
$$

and evaluate it at a temperature $T$ just above $T_{c}$. This imaginary-axis extrapolation procedure is robust in producing a scaling result even if the system is not a Fermiliquid, where $1 / Z(0)$ would measure the quasiparticle weight. For example, in the classical-phonon case, the lowest-order contribution to the self energy is $\Sigma(z)=$ $-T U G(z)$. The curvature of $\operatorname{Im} \Sigma(\omega)$ at the chemical potential is positive. Hence the system is never a Fermi liquid, except at $T=0$, where it becomes noninteracting. Nevertheless, the extrapolation procedure given in Eq. (3) still falls on the scaling curve, and for the weakly-coupled classical-phonon case, it produces $Z(0)=$ $1+4|U| \rho(0) / 3$, rather than the expected result of $Z(0)=$ $1+|U| \rho(0)$ from Migdal-Eliashberg theory. We find that a phenomenological functional form that fits the data relatively well is $T_{c}=0.182 Z(0)^{-0.12} \exp [-0.75 /\{Z(0)-1\}]$ (the solid line in Fig. 2).

As a consequence of this scaling, if we use the quasiharmonic approximation to describe an anharmonic system with the parameters adjusted to obtain correspondence in $Z(0)$, then we also accurately reproduce two-particle properties like $T_{c}$. Since this result is robust against introducing anharmonicity, we believe this is the reason why the quasiharmonic approximation works so well in real materials.

We plot the interacting density of states and the self energy in Figs. 3-5 for weak-coupling harmonic and anharmonic cases, and for a strong-coupling harmonic case. We range from a high temperature down to just above $T_{c}$. Notice how the weak-coupling cases are not Fermi liquids, and have quite different DOS and self energies, 
but both map onto the same $Z(0)$ and $T_{c}$ [our definition of $Z(0)$ from the imaginary axis is not equal to the derivative of the self energy on the real axis, which has the opposite sign for a Fermi liquid here]. Furthermore, the paramagnetic phase of this model becomes noninteracting as $T \rightarrow 0$ since $\Sigma$ vanishes in the weak-coupling limit, which also explains why the anharmonic DOS becomes more symmetric as $T \rightarrow 0$. Scaling even holds (in a more approximate way) when the system is a bipolaronic insulator as shown in Fig. 5-the self-energy has a large imaginary part near the chemical potential, which grows as $T$ is lowered, and a pseudogap develops in the interacting density of states. This is then followed by a gap developing at lower $T$ and the imaginary part of the self-energy vanishing within the gap region except for a narrow spike at the frequency where the real part of the self-energy changes sign. But the $Z(0)$ parameter can still be defined on the imaginary axis!

Conclusion We have discovered an approximate scaling relation for the electron-phonon problem that holds over a wide range of phonon frequencies, coupling strengths, and anharmonicities. It relates the wavefunction renormalization parameter, extracted from the imaginary axis, to the transition temperature. Our results show that anharmonic models that are tuned to the same $Z(0)$ will show similar $T_{c} \mathrm{~s}$ as harmonic models with the same $Z(0)$. We feel this underlying scaling behavior helps explain the success of the quasiharmonic approximation employed in describing nearly all real materials.

We would like to acknowledge useful conversations with J. Hirsch, G. Mahan, P. Miller, B. Schüttler, and J. Serene. J. K. F. acknowledges support of ONR grant yip-n000149610828 and J. K. F. and V. Z. acknowledge support of NSF grants DMR-9627778 and INT-9722782. M. J. acknowledges support of NSF grants DMR-9704021 and DMR-9357199. Supercomputer time was provided by grant DMR950010P from the Pittsburgh Supercomputing Center, sponsored by the NSF.

[1] G. P. Srivastava, The Physics of Phonons (Adam Hilger, Bristol, 1990).

[2] A. B. Migdal, Zh. Eksp. Teor. Fiz. 34, 1438 (1958) [Sov. Phys.-JETP 7, 999 (1958)].

[3] G. M. Eliashberg, Zh. Eksp. Teor. Fiz. 38, 966 (1960) [Sov. Phys.-JETP 11, 696 (1960)].

[4] J. C. K. Hui and P. B. Allen, J. Phys. F 4, L42 (1974); A. E. Kavakozov and E. G. Maksimov, Zh. Eksp. Teor. Fiz. 74, 681 (1978) [Sov. Phys. JETP 47, 358 (1978)]; G. D. Mahan and J. O. Sofo, Phys. Rev. B 47, 8050 (1993).

[5] J. K. Freericks, M. Jarrell, and D. J. Scalapino, Phys. Rev. B 48, 6302 (1993); J. K. Freericks, M. Jarrell, and D. J. Scalapino, Europhys. Lett. 25, 37 (1994); J. K. Fre- ericks and M. Jarrell, in Computer Simulation Studies in Condensed Matter Physics VII, edited by D. P. Landau, K. K. Mon, and H.-B. Schüttler, (Springer-Verlag, 1995); J. K. Freericks and M. Jarrell, Phys. Rev. Lett. 75, 2570 (1995); J. K. Freericks, M. Jarrell, G. D. Mahan, Phys. Rev. Lett. 77, 4588 (1996); Erratum: Phys. Rev. Lett. 79, 1783 (1997)

[6] J. E. Hirsch, Phys. Rev. B 47, 5351 (1993); F. Marsiglio and J. E. Hirsch, Phys. Rev. B 49, 1366 (1994).

[7] W. Metzner and D. Vollhardt, Phys. Rev. Lett. 62, 324 (1989).

[8] J. E. Hirsch and R. M. Fye, Phys. Rev. Lett. 56, 2521 (1986).

[9] U. Brandt and C. Mielsch, Z. Phys. B 75, 365 (1989); 79, 295 (1990); 82, 37 (1991).

[10] A. J. Millis, P. B. Littlewood, and B. Shraiman, Phys. Rev. Lett. 74, 5144 (1995); A. J. Millis, R. Mueller, and B. Shraiman, Phys. Rev. B 54, 5389 (1996).

[11] T. Holstein, Ann. Phys. 8, 325 (1959).

[12] M. Jarrell, Phys. Rev. Lett. 69, 168 (1992).

[13] J. K. Freericks, Phys. Rev. B 50, 403 (1994).

[14] J. K. Freericks, Phys. Rev. B 48, 3881 (1993); J. K. Freericks and G. D. Mahan, Phys. Rev. B 54, 9372 (1996); Erratum: Phys. Rev. B 56, 11321 (1997).

FIG. 1. Transition temperatures for the chessboard phase charge-density-wave order at half filling. (a) $T_{c}$ for the harmonic case. The horizontal axis is the effective electron-electron coupling $|U|=g^{2} / \kappa$. Three frequencies are included $\Omega=0, \Omega=0.5$, and $\Omega=\infty$. The dashed line is a conserving second-order perturbative approximation (including vertex corrections) and the solid line is a second-order strong-coupling approximation. (b) $T_{c}$ for the anharmonic case. The horizontal axis is a measure of the anharmonic contribution to the energy (as described in the text). Open symbols are QMC simulations at $\Omega=0.5$, while solid symbols are exact solutions at $\Omega=0$.

FIG. 2. Scaling curve for the anharmonic phonon problem. The horizontal axis is the imaginary-axis-extrapolated wave-function renormalization parameter, extrapolated at $T_{c}$, while the vertical axis is the CDW $T_{c}$ at half filling. Included in the curve are weak-coupling results for the classical phonons with (dotted) vertex corrections, a conserving second-order approximation for the $\Omega=\infty$ case (dashed), and the approximate form described in the text (solid).

FIG. 3. Interacting density of states for a generic weak-coupling harmonic case $(\Omega=0, g=0.625$ and $\left.\alpha_{a n}=0.0\right)$ for six temperatures running from top to bottom at $\omega=0 T=0.04,0.0625,0.125,0.25,0.5,1.0$, and 2.0. The real and imaginary parts of the self energy are plotted in the insets. Notice how the slope of the real part of $\Sigma$ has the wrong sign, and how the imaginary part has a local minimum at the chemical potential, signifying that this system is not a Fermi liquid. 
FIG. 4. Interacting density of states for a generic weak-coupling anharmonic case $(\Omega=0, g=2.5$ and $\left.\alpha_{a n}=4.5\right)$ for the same six temperatures shown in Figure 3. Notice how the density of states is now asymmetric at high $T$ but becomes more symmetric as $T$ decreases.

FIG. 5. Interacting density of states for a generic strong-coupling anharmonic case $(\Omega=0, g=2.5$ and $\left.\alpha_{a n}=0.004\right)$ for six temperatures running from top to bottom (at $\omega=0) T=0.075,0.125,0.25,0.5,1.0$ and 2.0. Notice how the density of states develops a pseudogap at the chemical potential, which then becomes a true gap as $T$ is lowered.

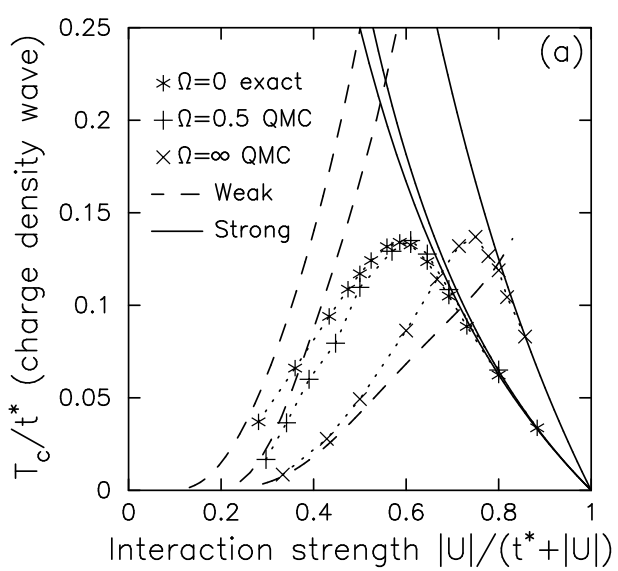

Freericks, Jarrell, and Zlatic, Phys. Rev. Lett., Figure 1(a)

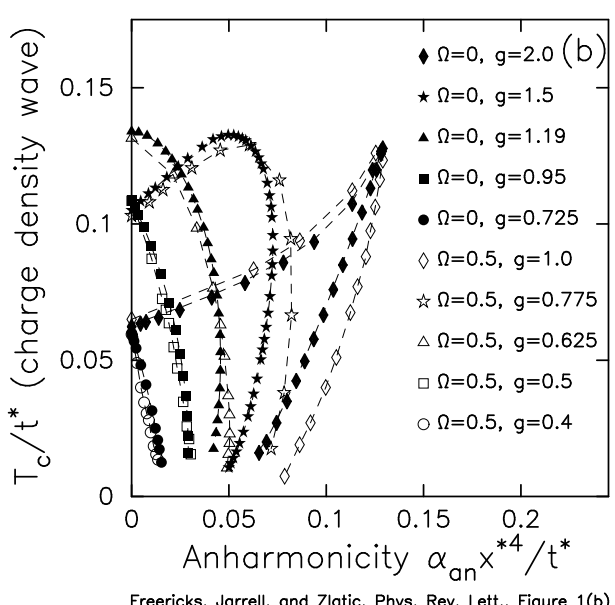

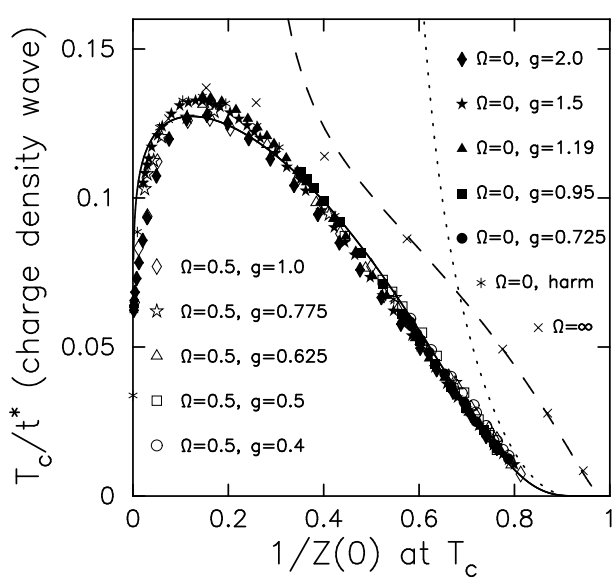

Freericks, Jarrell, and Zlatic, Phys. Rev. Lett., Figure 2

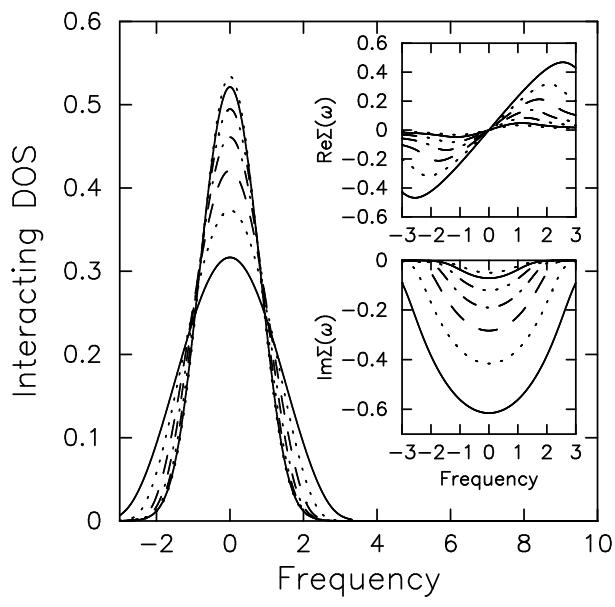

Freericks, Jarrell, and Zlatic, Phys. Rev. Lett., Figure 3

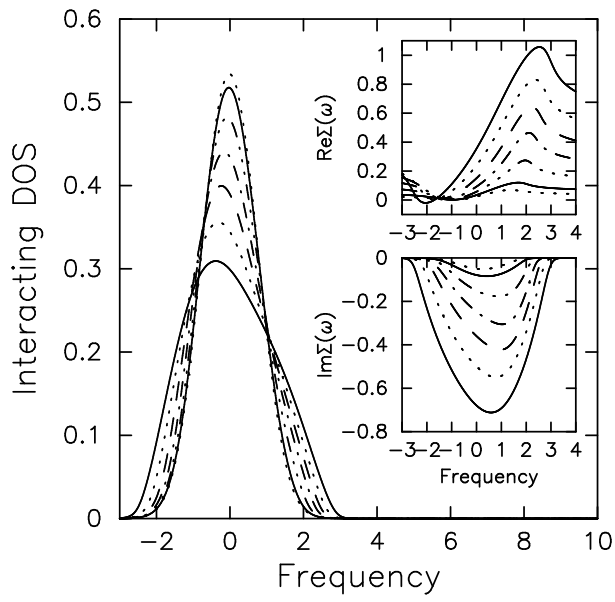

Freericks, Jarrell, and Zlatic, Phys. Rev. Lett., Figure 4 


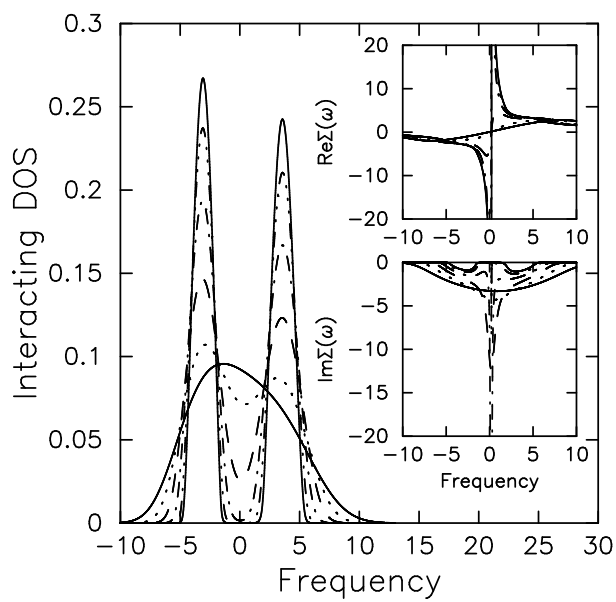

Freericks, Jarrell, and Zlatic, Phys. Rev. Lett., Figure 5 